\title{
Tyrer-Cuzick Model
}

National Cancer Institute

\section{Source}

National Cancer Institute. Tyrer-Cuzick Model. NCI Thesaurus. Code C91783.

A breast cancer risk model that incorporates both genetic and nongenetic factors. It uses a combination of extensive family history, endogenous estrogen exposure, and benign breast disease (atypical hyperplasia) in order to predict a 10-year risk of breast cancer development. (Amir, Evans, Shenton, et al., www.jmedgenet.com) 